\title{
PROPUESTA DE UN PROGRAMA DE INTELIGENCIA EMOCIONAL PA- RA MENORES INFRACTORES
}

\section{Tara Guerra González}

\section{Resumen}

El presente trabajo hace un recorrido por los modelos teóricos más importantes de Inteligencia Emocional (IE), con intención de dar una visión amplia sobre este concepto y finalmente se ha centrado en dos de ellos, el modelo de Mayer y Salovey (1997) y el modelo de Goleman (1996), así como por los instrumentos de medida de IE y otros programas que aborden el mismo propósito que el que aquí se presenta: la mejora de la IE. Concretamente se hace una propuesta de intervención en menores infractores para la mejora de la IE. Por tanto, se aborda también la situación concreta de la Justicia Juvenil en España y un análisis breve de los factores implicados en la delincuencia juvenil. Por último, se presenta el programa, que consta de 7 sesiones basadas en las 4 dimensiones del modelo de Mayer y Salovey (1997) y con una sesión basada en el concepto de empatía de Goleman (1996), distribuidas en 8 semanas.

\section{Palabras clave:}

inteligencia emocional; Mayer y Salovey; menores infractores; programa de intervención.

\begin{abstract}
The present work make a tour of the most important theorical models of Emotional Intelligence (EI), with the intention of giving a broath vision on this concept and finally has focused of two of then, the Mayer an Salovey's (1997) model and the Golleman's (1996) model, as well as by the EI measurement instruments and others programs that address the same purpose as the one presented here: the improvement of EI. Specifically a proposal in made for intervention in minor offenders to improve EI. Therefor, it also adresses the specific situation of Juvenil Justice in Spain and a brief análisis of the factors involved in juvenil delinquency. Finally, the program is presented, which consists of 7 sessions based on the 4 dimentions of the Mayer and Salovey model (1997) and one session based on Golleman's concept of empathy (1996), distributed over 8 weeks
\end{abstract}

\section{Keywords:}

emotional inteligence; Mayer and Salovey; minors offenders; program of intervention
Datos del autor

TARA GUERRA GONZÁLEZ

Correspondencia: taraguegon@gmail.com

\author{
UNIVERSIDAD DE AlMERÍA \\ Trabajo Fin de Grado \\ Grado en Psicología
}

Curso 2019-2020 
Introducción

En el año 1996 el psicólogo Daniel Goleman escribe un libro titulado Inteligencia Emocional (IE) con la intención de difundir este concepto en la sociedad (Goleman, 1996), sin saber que el impacto mediático de estas primeras publicaciones serían las que catapultarían este constructo psicológico al punto de mira de estudiosos, investigadores y científicos (Sánchez-Teruel y Robles-Bello, 2018)

El constructo IE fue esbozado por Peter Salovey y John Mayer (1990), en un momento histórico en el que el Cociente Intelectual (CI) era la única medida aceptada en la comunidad científica, como una forma objetiva de medir la inteligencia de una persona.

En el campo de la Psicología ha comenzado a hacerse uso de la IE para abordar diferentes ámbitos de intervención, en el área de educación (Avilés, 2005; Barchard, 2003), en salud (Berrios Martos et al., 2006; Bisquerra, 2000), en bienestar y felicidad (Extremera et al., 2011; Fernández-Berrocal y Extremera, 2009), en el ámbito empresarial (Caruso y Salovey, 2005; Goleman, 1996, 1999) y en el campo científico (Bar-On y Parker, 2000; Mayer y Salovey, 1993, 1997).

Modelos teóricos de la IE

Mayer et al. (2000) hacen una distinción entre dos tipos de modelo de IE: por un lado, se sitúan los modelos mixtos o de rasgo, en el que la IE se concibe como una mezcla de habilidades con características de personalidad.

Goleman (1996) desarrolla su propio modelo y define la IE como la "capacidad de reconocer los propios sentimientos y los de los demás, de motivarnos y de manejar adecuadamente las relaciones" y que ha ido evolucionando con el tiempo, culminando en la conclusión de que este concepto se encuentra formado por cuatro dimensiones compuestas por diferentes competencias (Goleman, 2011).

Dentro de los modelos mixtos, también se encuentra el modelo de Bar-On, (2006) llamado ESI (Emotional Social Intelligence), en el que se define la IE como "una muestra representativa de las competencias, habilidades y facilitadores sociales y emocionales que determinan la efectividad de entendernos y expresarnos con nosotros mismos, como entendemos y nos relacionamos con los otros y lidiamos con las demandas diarias".

Por otro lado, se encuentran los modelos teóricos que hacen referencia a la IE como habilidad, que se centra en el procesamiento emocional de la información y en el estudio de las capacidades que se encuentran relacionadas con dicho procesamiento (Gázquez y cols.,

2015). En esta línea, para Mayer y Salovey (1997) en su modelo de habilidades, la IE es "la capacidad para supervisar los sentimientos y las emociones de uno mismo y de los demás, de discriminar entre ellos y usar esta información para la orientación de la acción y el pensamiento propio".

Salovey y Mayer (1990), revisando una serie de trabajos de investigación, iniciaron el camino para dar forma a un marco conceptual de la IE. En su modelo , Mayer y Salovey (1997) distinguen entre cuatro elementos que componen la Inteligencia emocional:

Percepción y expresión de las emociones: es la habilidad más básica y hace referencia a la certeza para identificar emociones y contenido emocional de las personas, tanto en sí mismas como en otras. Incluye el registro, la atención y la identificación de los mensajes emocionales, su manifestación se observa en expresiones faciales, movimientos corporales, posturas, tonos de voz, etc. Se conforma de cuatro subhabilidades: a) habilidad para identificar emociones en las sensaciones, estados físicos y pensamientos propios, b) habilidad para identificar emociones en otras personas, formas de arte, lenguaje, sonidos, apariencia y comportamiento, c) habilidad para expresar emociones adecuadamente y para expresar necesidades relacionadas con esos sentimientos y d) habilidad para discriminar la adecuada o inadecuada, honesta o deshonesta expresión de los sentimientos (Mayer y Salovey, 1997).

Facilitación emocional del pensamiento: establecida como poder hacer uso de las emociones como parte de los procesos cognitivos, debido a que nuestras emociones dirigen la atención hacia cierta información "relevante", determinando así la manera en que la procesamos y la forma en la que enfrentamos los problemas. Compuesta de cuatro subhabilidades: a) priorización y redirección del pensamiento basado en los sentimientos, b) uso de emociones para facilitar el juicio y la memoria, c) capitalización de variaciones en los estados emocionales para permitir al individuo cambiar de perspectiva y considerar diversos puntos de vista y d) uso de diferentes estados emocionales para facilitar formas específicas de abordar un problema, el razonamiento y la creatividad (Mayer y Salovey, 1997).

Comprensión emocional: es decir, el conocimiento de cómo funciona nuestro sistema emocional a nivel cognitivo y la forma en que afectan las emociones a los procesos racionales. Ser capaces no sólo de etiquetar las emociones simples y complejas, sino también saber que puede pasarse de un estado emocional a otro. Igual que las otras ramas contiene cuatro subhabilidades: a) habilidad para etiquetar emociones y reconocer las relaciones entre ellas y las palabras, como las relaciones entre gustar y amar, b) habilidad para la expresión de las emociones en función de relaciones, por ejemplo que la tristeza siempre acompaña a una pérdida, c) habilidad para entender emociones complejas como sentimientos simultáneos de amor y odio o combinaciones de amor y tristeza y d) la habilidad para reconocer posibles transiciones entre emociones como el paso de enojo a satisfacción o tristeza (Mayer y Salovey, 1997).

Regulación reflexiva de las emociones: esta habilidad consiste en estar abierto a estados de ánimo emocionales de todo tipo, reflexionando sobre ellos y saber si la información que los acompaña es útil o no, sin reprimirla ni exagerarla. Además de ser capaces de regular las propias emociones y las ajenas. La habilidad de regulación reflexiva se compone de cuatro subhabilidades: a) habilidad para permanecer abiertos a sentimientos, ya sean placenteros o no, b) habilidad para atraer o distanciarse reflexivamente de una emoción dependiendo de su información o utilidad juzgada, c) habilidad para monitorear reflexivamente las emociones en uno mismo y otros reconociendo cuán puras, comunes, importantes o razonables son y d) la habilidad de manejar en uno mismo y otros las emociones, incrementando sus efectos negativos y maximizando los positivos sin reprimirlas o exagerarlas al momento de expresarlas (Mayer y Salovey, 1997).

En su modelo tratan de recoger claramente que la IE se compone de habilidades de procesamiento de la información, no de competencias. Señalando que es un instrumento que evita que el comportamiento sea etiquetado de "bueno" o "malo", sino que señala la dirección para el proceso de investigación personal respetando los elementos del entorno cultural, político y religioso, así como otras características personales del individuo (Mayer y Salovey, 1997).

En su modelo, Goleman (2011) también considera que la IE se encuentra integrada por cuatro dimensiones que se componen de diversas competencias: 1. El conocimiento de uno mismo. Dimensión conformada por la competencia del autoconocimiento emocional, que a su vez comprende las capacidades de atender las señales internas; reconocer como los propios sentimientos afectan al desempeño laboral; escuchar la intuición y hablar abiertamente de las emociones como guía de las propias acciones; 2 . La autorregulación, estaría relacionada como el manejo del mundo interno para beneficio propio, o de los demás, y se compone de las competencias de autocontrol emocional, orientación a los resultados, adaptabilidad y optimismo; 3 . La conciencia social, cuyas competencias, la empatía y la conciencia organizacio- 
nal son esenciales para establecer buenas relaciones interpersonales; 4 . La regulación de las relaciones interpersonales. Es la capacidad relacionada con los aspectos de persuasión e influencia sobre otros/as y está formada por las competencias de inspiración al liderazgo, influencia, manejo del conflicto y trabajo en equipo y colaboración (American Management Association, 2012; Goleman,2011). Instrumentos para medir IE

Trait Meta-Mood Scale (T.M.M.S.) diseñada por Salovey et al. (1995) y adaptada al español por Fernández-Berrocal et al. (2004), pasó de tener 48 ítems a tener 24 en la adaptación al español. Se compone de tres escalas: 1) Atención a los sentimientos (nivel de creencia sobre la focalización emocional); 2) Claridad emocional

(percepción subjetiva de las propias emociones); 3) Reparación de las emociones (creencia de poder interrumpir y regular los estados emocionales negativos y potenciar los positivos), con respuestas tipo Likert de 5 puntos y para edades entre 18 y 57 años. Decir también, que se trata de una escala autoinformada.

Mayer-Salovey-Caruso Emotional Intelligence Test (MSCEIT v.2.0.) desarrollado por Mayer et al., (2002) y con adaptación al castellano por Extremera y cols. (2002), es una versión más corta del Mayer-Salovey-Caruso Emotional Intelligence Test (Mayer et al.,

2001) de 141 ítems que miden los cuatro aspectos del modelo original de Mayer y Salovey (1997) para población de entre 16 y 58 años. Y presenta propiedades psicométricas aceptables y ha sido comprobada su idoneidad para la aplicación en hispano hablantes (Extremera et al., 2006). Esta por su parte, es una escala de ejecución.

Emotional Quotient Inventory (EQ-i) desarrollado por BarOn (1997) siendo uno de los primeros instrumentos para medir la IE, incluyendo aspectos afectivos y sociales consta de 133 ítems que evalúan los 5 componentes de su modelo ESI con un total de 15 subescalas en formato tipo Likert de 5 puntos $(1=$ de acuerdo, $5=$ desacuerdo) y destinada a personas de 6 a 17 años. Sin embargo, Bar-On (2002) desarrolló una versión más corta de 51 ítems (EQ-i: Short) con 5 subescalas de respuesta tipo Likert de 5 puntos $(1=$ nunca, $5=$ siempre). Es una escala autoinformada y está adaptada al español por López-Zafra et al. (2014).

IE, su relación con otras variables y la adolescencia

Pasando al papel que tiene la IE en la sociedad, debe resaltarse su especial importancia en la adolescencia, debido a que en este período de tránsito de infante a adulto hay una sucesión de cambios hormonales que conllevan consigo un descontrol de las emociones (Sánchez, 2009). En esta etapa del desarrollo vital, tienen lugar una serie de reajustes psicológicos en todas las esferas de la vida: familiar, amistades, personas adultas de fuera de la familia y en relación con el propio sentido de la identidad (Coleman y Hendry, 2003). En esta época de cambios, es posible que aquellos adolescentes con mejor habilidad a la hora de saber cómo se sienten las personas de su entorno, utilicen dicha información no sólo para mostrar una mayor empatía hacia ellos, o para ayudarles a regular las emociones que sienten, sino también para modificar su comportamiento y adecuarse mejor a las situaciones sociales (Salguero et al., 2011).

Ciarrochi et al., (2001) demostraron que los adolescentes con alta IE eran capaces de establecer y mantener relaciones interpersonales. Tenían más amigos o mayor cantidad de apoyo social, sentían mayor satisfacción con las relaciones establecidas a nivel social, poseían más habilidades para identificar expresiones emocionales y presentaban comportamientos más adaptativos para mejorar sus emociones negativas cuando se controlaban los efectos de otras variables psicológicas como autoestima o ansiedad.

Se ha relacionado la IE con una mejor autoestima (Romero et al., 2017), los procesos motivacionales (Cera et al., 2015) y las conductas prosociales (Gázquez et al., 2015). García-Sancho et al. (2014) realizaron una revisión sistemática de 19 estudios, 12 contenían información que relacionaba la conducta agresiva con la inteligencia emocional. A partir de los cuales se extrajo la conclusión de que los/as niños/as con mayores puntuaciones en EI, presentaban menor número de conductas agresivas, menores niveles de acoso escolar y una mayor cantidad de conductas prosociales hacia sus compañeros/as de clase.

En el estudio realizado por Zavala et al. (2008) se encontraron correlaciones significativas entre los factores de la IE y las habilidades sociales, que ponen de manifiesto la relación que existe entre estos constructos, encontrando una correlación positiva entre ambas variables y apoyando la línea de investigación empírica que trata de establecer la IE como una variable clave para el mantenimiento de las interacciones sociales positivas.

Delgado-Gómez et al. (2019) encontraron diferencias significativas en la puntuación obtenida por estudiantes universitarios en diferentes ramas de IE autopercibida y la presentación de sintomatología psicopatológica, predominando la relación entre la capacidad de prestar más atención a los sentimientos y sintomatología depresiva; la capacidad de comprender bien los estados emocionales y reparar el estado de ánimo con la mayoría de las escalas clínicas. A medida que aumentaba la capacidad de comprender los estados emocionales, aumentaba la capacidad de regular y/o reparar el estado de ánimo.

\section{Programas de IE en adolescentes}

En 2011, Redondo et al. elaboraron un informe de investigación para analizar los factores de éxito asociados a los programas de intervención en menores infractores, recogiendo que aquellos menores que eran sometidos a un programa de intervención reincidían un $44 \%$ frente al $53,5 \%$ de los jóvenes que no recibían tratamiento específico. En este mismo estudio, se recogió que las intervenciones orientadas a entrenar habilidades de vida $(\mathrm{r}=0,18)$ tenían la eficacia más alta entre las diferentes categorías establecidas para diferenciar el tipo de intervención.

Existen numerosos programas de IE diseñados de forma específica o adaptables a adolescentes. Entre ellos se encuentra POCOSE (Repetto et al., 2007), basado en el modelo de Bar-on (2006) y el de Goleman (1999), y dirigido a estudiantes de último ciclo de secundaria y universitarios. Además, consta de siete módulos que se desarrollan en 5 sesiones de dos horas y media para desarrollar las competencias de autoconocimiento, empatía, regulación, resolución de conflictos, trabajo en equipo, asertividad y motivación.

Otro programa diseñado para desarrollar la IE es el que aportan Ramos et al. (2012) basándose en el uso de herramientas de Mindfulness, diseñado para adultos, pero con posibilidad de adaptación desde primaria hasta la universidad. Su eficacia ha sido probada en educación primaria pero no en educación secundaria (Ponce, 2016).

Uno de los mejores programas educativos en IE con eficacia demostrada en adolescentes es el programa INTEMO, desarrollado por Noguera et al. (2014) en el laboratorio de las emociones de la Universidad de Málaga, se basa en el modelo de Mayer y Salovey (1997) para abordar el aprendizaje de las habilidades la IE y consta de un total de 12 sesiones. Ha demostrado ser útil para promocionar los procesos de afrontamiento emocional y ampliar el abanico de habilidades emocionales basadas en la comprensión manejo y regulación de estados emocionales (Díazet al., 2020).

\section{Justicia Juvenil en España}

Actualmente en España, las directrices que enmarcan la justicia juvenil vienen recogidas en la Ley Orgánica 5/2000 de 12 de enero, reguladora de la responsabilidad penal de los menores. En ella se refleja la naturaleza reeducativa e individualizada, teniendo en cuenta las circunstancias 
familiares, sociales y de personalidad de los menores infractores. La responsabilidad penal de menores en España se exige a las personas mayores de catorce años y menores de dieciocho por la comisión de hechos tipificados como delitos o faltas en el Código Penal o en las leyes penales especiales.

Los factores que influyen en la realización de las conductas tipificadas como hechos delictivos por parte de los/as menores son los factores de riesgo y factores de protección. Los factores de riesgo son aquellas variables que incrementan la probabilidad de la conducta antisocial penada, mientras que los factores de protección son los que disminuyen la probabilidad de comisión de comportamientos desviados (Vázquez et al., 2003). Los factores de riesgo pueden ser estáticos o dinámicos. Los factores de riesgo estáticos no son susceptibles de modificación. En cambio, los factores de riesgo dinámicos son aquellos que pueden ser mejorados con las intervenciones adecuadas (Redondo et al., 2011).

Alguna de las características psicológicas individuales que parecen facilitar el acto delictivo son un cuadro clínico asociado a trastornos de conducta, un alto grado de impulsividad y déficit en competencias sociales o emocionales (Sanabria y Uribe, 2010). En esta línea, un estudio realizado por Vilariño et al. (2013) encontró diferencias significativas entre menores infractores y no infractores en competencias y destrezas sociales, concretamente, los menores infractores presentaban limitaciones en IE, entre otras. Así mismo, se sugiere el diseño e implementación de programas específicos en estas áreas sociales que permitan ayudar a la reinserción de los/as jóvenes. Otros autores apuntan que algunas medidas, buscando una mejora prosocial podrían contemplar el uso de talleres de entrenamiento de habilidades sociales, control de la impulsividad, resolución de conflictos o promoción de la inteligencia emocional (Buil-Legaz et al., 2019).

Además de los factores de riesgo y de protección como piedra angular para predecir la probabilidad de que se cometa un delito por parte de un/a menor, se debe tener en cuenta la historia delictiva previa como indicador para prevenir la futura reincidencia de los menores infractores (Capdevila et al., 2005). El uso de programas de intervención en este colectivo, como lo que se plantea en este manuscrito, en centros o instituciones que pertenezcan al Sistema Judicial de Menores, debería estar orientado principalmente a disminuir la probabilidad de reincidencia de los menores.

Objetivo

El objetivo del presente trabajo es desarrollar un Programa de IE basado en el Modelo de Mayer y Salovey (1997) para aplicarlo en menores infractores.

Método

Participantes

El programa va dirigido a personas de entre 14 años y 21 que se encuentren bajo medida judicial en el sistema de justicia juvenil y necesiten mejorar sus competencias en gestión y percepción de las emociones propias y ajenas.

Instrumentos

MSCEIT v. 2.0. versión española (Extremera et al., 2002). Adaptación del MayerSalovey-Caruso Emotional Intelligence Test v.2.0. (Mayer et al., 2002) mediante un proceso de traducción directa y traducción inversa; y posterior aplicación en una muestra de 946 estudiantes universitarios de entre 16 y 58 años. Obteniendo buenos niveles de fiabilidad (entre 0,63 y 0,90 ) y consistencia interna. Consta de 141 ítems comprendidos en 8 subescalas, dos para cada dimensión del modelo de Mayer y Salovey (1997) y un tiempo de ejecución de entre 30 y 45 minutos.

Hoja de observación participante (Anexo 1). Elaborada ad hoc. Con intención de aportar información sobre el comportamiento relevante de cada participante durante el desarrollo de las sesiones, que puede ser útil para valorar cambios en las dinámicas o para informes internos de la institución que implemente el programa. Consta de una tabla, con tres áreas que evalúan los conocimientos previos que parece mostrar el/a participante: entendida como "alta" cuando el/a participante verbalice en varias ocasiones elementos relacionados con el objetivo de la sesión, "media" cuando solo lo haga en alguna ocasión y "baja" cuando no los verbalice en ninguna ocasión. El interés mostrado durante el desarrollo de la sesión: entendido como "alta" cuando la atención prestada a las explicaciones que da el/a monitor/a se extienda durante toda la sesión, "media" cuando dicha atención se pierda en más de tres ocasiones y "baja. Y el grado de participación en la misma: entendido como "alta" cuando interaccione en todas las actividades de forma activa, "media" cuando sólo lo haga en alguna y "baja" cuando lo haga en una o ninguna.

Cuestionario breve de evaluación (Anexo 2). Elaborado ad hoc. Con la finalidad de conocer de manera escrita el alcance de los contenidos a nivel individual. Consta de dos ítems de pregunta abierta y tres de opción múltiple (dos de ellos de respuesta dicotómica y uno con escala tipo Likert de 4 opciones).

Cuestionario de Satisfacción (Anexo 3). Elaborado ad hoc. Para evaluar el grado de satisfacción con el programa y sus contenidos por parte de los participantes. Consta de seis ítems, tres de elección múltiple (dos dicotómicos y uno de escala tipo Likert de diez opciones, siendo 1 nada y 10 muchísimo) y tres de pregunta abierta.

Cuestionario para la mejora del programa (Anexo 4). Elaborado ad hoc. Con intención de recibir información útil para mejorar el programa a partir de las consideraciones y aportaciones de los/as monitores/as que lo lleven a cabo. Consta de tres ítems: uno de ellos es una tabla que recoge las ocho sesiones y que indiquen el grado en que consideran adecuados los contenidos de cada sesión con una escala tipo Likert donde $0=$ nada adecuados y $5=$ muy adecuados. Otro es una pregunta abierta para comunicar posibles problemas/áreas a mejorar durante el desarrollo del programa. Y, por último, una tabla de tres áreas (claridad de los contenidos, idoneidad del formato y utilidad de las actividades) de respuesta en escala tipo Likert donde 1 = nada satisfecho/a y $5=$ muy satisfecho/a.

Propuesta de programa de intervención en IE Objetivos del programa

El objetivo principal de este programa será que las personas destinatarias del mismo incrementen el nivel de Inteligencia Emocional, beneficiando su reeducación emocional y el desarrollo de habilidades emocionales que les permitan adquirir mayor autocontrol, autoconocimiento y mejorar sus relaciones intra e interpersonales.

Los objetivos específicos del programa de intervención son:

Comprender el concepto de inteligencia emocional y las emociones básicas y complejas.

Potenciar el conocimiento sobre empatía y activar mecanismos de empatía.

Mejorar la expresión de emociones y las necesidades asociadas a ellas.

Facilitar la exposición de experiencias personales en presencia de un grupo.

Incentivar la activación de mecanismos para la comprensión emocional.

Mejorar el reconocimiento de emociones propias y de otros/as.

Aprender técnicas para facilitar el pensamiento por medio de las emociones (memoria, razonamiento y creatividad).

Hacer uso de las emociones para abordar problemas y mejorar el razonamiento.

Aumentar la capacidad de autocontrol de las emociones. 
Hacer una reflexión sobre los comportamientos emocionales propios y de otros/as.

\section{Temporalización}

El programa cuenta con un total de 8 sesiones que estarán distribuidas en 8 semanas, con una duración de una hora y media por sesión aproximadamente.

\section{Estructura del Programa}

Aunque el programa está pensado para su aplicación grupal en pequeños grupos (máximo 7 personas por sesión), si el/la psicóloga que lo va a aplicar lo considera oportuno, puede impartirlo de manera individual. Todas las sesiones tendrán un formato similar que corresponde con trabajo en grupo en la sesión por medio de una presentación de Power Point donde se expondrán datos y se hará un abordaje algo más teórico sobre los contenidos de cada una. Se hará una actividad práctica relacionada con el contenido de la sesión y por último se prescribirá una tarea individual que deberán llevar a cabo de manera autónoma durante la semana y que se revisará en la siguiente sesión. Las presentaciones serán dinámicas y participativas, con poco texto y muy gráficas, para facilitar la asimilación de los conocimientos y mantener la atención de los/as participantes. El programa está diseñado para que se lleve a cabo por un/a psicólogo/a que dirija las sesiones, haga las evaluaciones y analice los resultados, aunque sería conveniente que durante las sesiones contara con una persona de apoyo para realizar las observaciones y tomar nota de ellas.

El soporte principal de las sesiones serán las presentaciones de Power Point que se adjuntan al programa. Es importante que se tome nota del comportamiento individual de cada participante ya que en el sistema Judicial Juvenil debe haber un seguimiento de cada caso para evaluar sus avances, sus cambios y sus posibles mejoras.

Deberá disponerse de una sala con sillas, donde se cuente con intimidad y se puedan desarrollar las sesiones en un ambiente tranquilo, que transmita a quienes participen una sensación de seguridad para expresarse y aprender con la menor interferencia posible. Los materiales principales de los que deberá hacerse uso son un cañón, una pantalla y un ordenador con acceso a internet, que sirvan de soporte para visualizar las presentaciones y los elementos audiovisuales.

Además, deberá hacerse uso de la observación participante, que incluye una serie de medidas que vienen recogidas en el Anexo 1, para establecer el grado de interés y participación de cada integrante del grupo, así como un cuestionario de satisfacción (Anexo 3) que deberá pasarse después de finalizar el programa y un cuestionario breve de evaluación (Anexo 2) que será utilizado tras cada sesión para conocer el alcance de los contenidos de cada sesión, para el que al comienzo de la primera sesión deberá otorgarse un código de 3 cifras de manera aleatoria a cada participante y de forma que sólo lo conozca cada participante. De esta forma se garantiza el anonimato en las opiniones, pero se permite evaluar a un mismo participante en sus opiniones para cada sesión.

Sesiones

*Evaluación previa por medio del MSCEIT de los/as participantes.

Lo primero que se hará es explicarles en qué consiste el programa y cómo se va a organizar (tabla 1). A continuación, se procederá a repartirles, de forma aleatoria, los códigos de 3 dígitos explicándoles que son sólo para controlar que no responden dos veces a un mismo cuestionario o que no responden ninguno, pero que el código sólo lo sabrá cada uno/a de ellos/as y deberán usarlo en todos los cuestionarios que respondan durante el programa, además de en las actividades escritas. También se les indicará que al finalizar el programa se le pedirá que digan su código y su nombre en otro cuestionario como este, con el único fin de obtener información útil para sus infor- mes. El uso del código es individual e intransferible y que se usará para que se sientan en el anonimato mientras se desarrolla el programa.

*Sesión 1: ¿Qué son las emociones?

Objetivo: comprender el concepto de inteligencia emocional (según el modelo elegido) y las emociones básicas y complejas.

Materiales/espacios: presentación Sesión 1. ¿Qué son las emociones?, cuestionario breve de evaluación (Anexo 2), plantilla de observación participante (anexo 1).

Procedimiento: se hará una breve exposición de la diferencia que existe entre emociones y sentimientos, cuáles son las emociones básicas y complejas, del erróneo etiquetado de las emociones como negativas/ positivas, de las características de intensidad y duración emocional y las actividades descritas a continuación. Al finalizar la sesión los/as participantes deben rellenar el cuestionario breve de evaluación (Anexo 2).

Actividad 1.1.

Video: presenta-

ción emociones

Objetivo: comen-

zar a conocer las

emociones bási-

cas.

Duración: 10 minutos aproximadamente.

Materiales: presentación de la Sesión 1 (diapositiva 3), video "Presentación las emociones".

Procedimiento: al llegar a la diapositiva $\mathrm{n}^{\circ} 3$, seguir el enlace para ver el video

"Presentación las emociones" de la cuenta de YouTube "Tere Gutiérrez" y comentar con los/as participantes el conocimiento que tienen sobre esas emociones y si conocen algunas más.

Actividad 1.2. Y tú, ¿qué sientes?

Objetivo: rememorar una situación y reconozcan la emoción que surgió durante esa experiencia, compartiéndolo con los/as compañeros/as de sesión.

Materiales: presentación de la Sesión 1 (diapositiva 8), papel y bolígrafo.

Duración: 20 minutos aproximadamente.

Procedimiento: Durante la sesión, cuando aparezca la diapositiva 8 , se le repartirá un papel y un bolígrafo a cada participante y se les pedirá que de manera anónima escriban una situación que les haya ocurrido recientemente que implicara alguna de las emociones que hemos visto. Cuando hayan finalizado se cogerán todos los papeles y se doblarán hasta que queden más pequeños y no se vea el texto escrito, se mezclarán aleatoriamente y se repartirán de nuevo para que lean en voz alta el papel que les ha tocado. Si alguien desea leer su papel podrá hacerlo como alternativa a la lectura anónima aleatoria.

Actividad 1.2. Video: El puente: inteligencia emocional" y debate

Objetivo: Reflexionar sobre el contenido del video y compartir su punto de vista sobre lo reflexionado con el resto de los/as participantes.

Duración: 20 minutos aproximadamente.

Materiales: presentación Sesión 1 (diapositiva 9), video "El puente: Inteligencia emocional".

Procedimiento: Durante la sesión, cuando aparezca la diapositiva 9, abrir el enlace para visualizar el video "El puente: Inteligencia emocional" del perfil de YouTube "JOHANNA BIJALBA" requerido para la realización posterior del debate acerca de lo observado en el mismo. Algunas preguntas que se podrán realizar para incentivar la participación son: ¿Les ha pasado alguna vez que se han visto en una situación similar a la del video?; ¿Qué hicieron en esa situación? ¿les hubiera gustado actuar de otra manera?; ¿Qué emociones han observado en los personajes del video?; ¿Qué personaje sale más perjudicado?; ¿por qué? 
* Sesiones 2 y 3: Correspondientes a la habilidad 1 del modelo teórico de Mayer y Salovey (percepción y expresión de las emociones). La sesión 2 tratará contenidos relacionados con las subhabilidades: a) habilidad para identificar emociones en las sensaciones, estados físicos y pensamientos propios, b) habilidad para identificar emociones en otras personas, formas de arte, lenguaje, sonidos, apariencia y comportamiento. La sesión 3 versará sobre las subhabilidades: c) habilidad para expresar emociones adecuadamente y para expresar necesidades relacionadas con esos sentimientos y d) habilidad para discriminar la adecuada o inadecuada, honesta o deshonesta expresión de los sentimientos.

Sesión 2. Reconocer las emociones.

Objetivo: Mejorar el reconocimiento de emociones propias y de otros/as.

Materiales: Presentación "Sesión 2. Reconocer las emociones", cuestionario breve de evaluación (Anexo 2), plantilla de observación participante (Anexo 1), una hoja de situaciones con emociones implicadas (Anexo 5) por cada participante.

Procedimiento: Se comenzará la sesión haciendo un pequeño repaso a lo aprendido en la anterior y exponiendo brevemente lo que se va a bordar durante esta segunda sesión. Al finalizar la sesión los/as participantes deben rellenar el cuestionario breve de evaluación (Anexo 2). Además, se llevarán a cabo las siguientes actividades:

Actividad 2.1. ¿Qué sentirías si...?

Objetivo: Rememorar/imaginar una situación y reconozcan la emoción que surgió durante esa experiencia, incluidos los síntomas físicos que experimentaron, compartiéndolo con los/as compañeros/as de sesión.

Duración: 15 minutos aproximadamente.

Materiales: Anexo 5.

Procedimiento: en esta dinámica deberá entregarse una hoja con situaciones emocionales a cada participante y que escriban la emoción que creen que sentirían si les ocurriera lo que se relata en cada situación. Posteriormente entregar al/a monitor/a el papel con sus respuestas para que las lea en voz alta y serán comentadas entre todos/as.

Actividad 2.2. Adivina qué emoción soy.

Objetivo: emparejar una emoción con sus síntomas físicos.

Duración: 10 minutos aproximadamente.

Materiales: diapositiva 5.

Procedimiento: cuando llegue la diapositiva ${ }^{\circ} 5$ aparecerán una serie de descripciones de síntomas físicos deben indicar con qué emoción la emparejarían. Después se comprobará con que emoción iba cada una de las descripciones.

Actividad 2.3. ¿qué estarán sintiendo?

Objetivo: identificar en base a la expresión facial las emociones

Duración: 10 minutos aproximadamente.

Materiales: diapositiva 11.

Procedimiento: cuando aparezca la diapositiva 11 deben tratar de reconocer las diferentes emociones que irán mostrando las caras. Actividad 2.4. Hasta los objetos sienten.

Objetivo: describir emociones humanas en objetos inanimados.

Duración: 5 minutos aproximadamente.

Procedimiento: en la diapositiva 12, aparecerán una serie de objetos inanimados que muestran una serie de rasgos similares a la expresión facial de las emociones humanas.

* Sesión 3. Expresar las emociones

Objetivo: Mejorar la expresión de emociones y las necesidades asociadas a ellas.

Facilitar la exposición de experiencias personales en presencia de un grupo.
Materiales: Presentación "Sesión 3. Expresar las emociones", cuestionario breve de evaluación (Anexo 2), plantilla de observación participante (Anexo 1)

Procedimiento: Se comenzará la sesión haciendo un pequeño repaso a lo aprendido en la anterior y exponiendo brevemente lo que se va a abordar durante esta sesión. Haciendo uso de la presentación "Sesión 3. Expresar las emociones" se procederá a explicar por qué es importante la expresión de las emociones de manera correcta y transmitir las necesidades que surgen con ellas a quienes nos rodean. También se llevarán a cabo las actividades descritas. Al finalizar la sesión los/as participantes deben rellenar el cuestionario breve de evaluación (Anexo 2).

Actividad 3.1. Video: expresión de las emociones.

Objetivo: aprender la importancia de la expresión emocional y que existen formas de hacerlo.

Duración: 15 minutos.

Materiales: diapositiva 5 de la presentación de la sesión 3, link del video "7

Beneficios de Expresar las Emociones"

Procedimiento: cuando aparezca la diapositiva 5, pondremos mediante el link en internet el video "7 Beneficios de Expresar las Emociones" de la cuenta de YouTube "Elena Emociones Básicas". Tras verlo se les preguntará que opinan sobre lo expuesto.

Actividad 3.2. Expresa lo que sientes y lo que necesitas.

Objetivo: Analizar las propias emociones y las necesidades que las pueden acompañar.

Duración: 15 minutos.

Materiales: presentación Sesión 3 (diapositivas 9 y 10).

Procedimiento: En una hoja de papel deben escribir como expresarían lo que sienten en las situaciones que se leerán en las diapositivas 9 y 10 . Es importante recordarles que no hay respuestas correctas, y que diferentes personas pueden sentir emociones distintas ante la misma situación. Después de escribir lo que sentirían y lo que necesitarían, entregan los papeles y se leerán en voz alta y se comentará entre todos/as si es adecuada la forma de expresar la emoción y las necesidades asociadas. Ofrecer la posibilidad de leer de forma voluntaria su papel en voz alta y luego entregarlo. Continuando la sesión se explica que es el lenguaje no verbal y su importancia en la detección de las emociones de los demás.

Actividad 3.3. Video: Lenguaje no verbal.

Objetivo: aprender aspectos del lenguaje no verbal.

Duración: 15 minutos.

Materiales: presentación Sesión 3 (diapositiva 17), video "Lenguaje NO VERBAL - La Guía de las MANOS"

Procedimiento: cuando aparezca la diapositiva $\mathrm{n}^{\circ} 17 \mathrm{se}$ seguirá el link del video "Lenguaje NO VERBAL - La Guía de las MANOS" de la cuenta de YouTube "Hemisferio derecho" y posteriormente se les preguntará que opinan sobre lo expuesto.

Actividad 3.4. Video: Las emociones y la voz

Objetivo: ver un ejemplo de la influencia de las emociones en el lenguaje no verbal.

Duración: 5 minutos.

Materiales: presentación Sesión 3 (diapositiva 18), video "Cómo influyen las emociones en nuestro tono de voz".

Procedimiento: cuando aparezca la diapositiva $n^{\circ} 18 \mathrm{se}$ seguirá el link del video

"Cómo influyen las emociones en nuestro tono de voz" de la cuenta de YouTube "Marta

Pinillos Manrique de Lara" y posteriormente se les pedirá que piensen en situaciones en que las emociones hayan podido influir en su lenguaje no verbal (el tono de voz o cualquier otro aspecto). 
* Sesiones 4 y 5 : correspondientes a la habilidad 2 del modelo teórico de Mayer y Salovey (facilitación emocional del pensamiento). La sesión 4 abordará las subhabilidades: a) priorización y redirección del pensamiento basado en los sentimientos, b) uso de emociones para facilitar el juicio y la memoria. Mientras que la sesión 5 , versará sobre las subhabilidades: c) capitalización de variaciones en los estados emocionales para permitir al individuo cambiar de perspectiva y considerar diversos puntos de vista y d) uso de diferentes estados emocionales para facilitar formas específicas de abordar un problema, el razonamiento y la creatividad.

\section{Sesión 4. Facilitación emocional. Parte 1}

Objetivo: Aprender técnicas para facilitar el pensamiento por medio de las emociones.

Materiales: Presentación "Sesión 4. Facilitación emocional. Parte 1", cuestionario breve de evaluación (Anexo 2), plantilla de observación participante (Anexo 1).

Procedimiento: Se comenzará la sesión haciendo un pequeño repaso a lo aprendido en la anterior y exponiendo brevemente lo que se va a bordar durante esta sesión. Haciendo uso de la presentación "Sesión 4. Facilitación emocional. Parte 1", comenzamos con una exposición de los componentes de la conducta (afectivo, cognitivo y comportamental). Al finalizar la sesión los/as participantes deben rellenar el cuestionario breve de evaluación (Anexo 2). También se llevarán a cabo las siguientes actividades:

Actividad 4.1. Video: Emoción y atención.

Objetivo: analizar cómo influye la emoción en nuestra atención.

Duración: 10 minutos.

Materiales: presentación Sesión 4 (diapositiva 4), video "La emoción condiciona la atención [Inteligencia Emocional]".

Procedimiento: en el momento que aparezca la diapositiva 4 , acceder al link del video

"La emoción condiciona la atención [Inteligencia Emocional]" de la cuenta de YouTube

"Miguel Ángel Diaz Escoto" y tras su visionado preguntar a los/as participantes que describan situaciones en las que su emoción ha interferido con su capacidad de concentración.

\section{Actividad 4.2. Dinámica.}

Objetivo: Analizar qué tipo de relación existiría entre lo que sentimos, pensamos y hacemos en varias situaciones.

Duración: 15 minutos.

Materiales: Presentación Sesión 4 (diapositivas de la 5 a la 9).

Procedimiento: A continuación (diapositivas 6 y 8), se van a poner una serie de situaciones que los/as participantes deberán tratar de imaginar y contestar en un papel, qué sienten, qué piensan y qué harían en esa situación primero la situación de la diapositiva 6 , Cuando acaben de escribir, se les mostrarán las respuestas de la diapositiva 7 y se pasará a la 8 para ver la siguiente situación, cuando acaben de escribir, mostrar las respuestas que se han puesto como las más probables en la diapositiva 9 y comprobar si han puesto algo parecido o no y preguntarles por qué.

Actividad 4.3. Video: El poder de las palabras y las emociones..." y debate.

Objetivo: Exponer opiniones sobre la influencia real de nuestras emociones en nuestro entorno y en nosotros/as mismos/as, compartiendo experiencias personales.
Duración: 15 minutos aprox.

Materiales: Presentación Sesión 4 (diapositiva 10), video "El poder de las palabras y de las emociones - Experimento del Arroz".

Procedimiento: en la diapositiva, pinchar el enlace para ver el video "El poder de las palabras y de las emociones - Experimento del Arroz" de la cuenta de YouTube "MÁGICAMente" que replica un experimento llevado a cabo por Emoto sobre la influencia de las emociones en partículas de agua, los/as participantes deberán expresar su opinión sobre el video y sobre experiencia personal relacionada con los efectos de las emociones en su vida o la de seres cercanos.

\section{Actividad 4.4. Experimento.}

Objetivo: Mostrar la influencia de las emociones en la capacidad de memoria.

Duración: 15 minutos aprox.

Materiales: Presentación Sesión 4 (diapositiva 11). Procedimiento: Se hacen dos grupos y se le entrega un papel a cada grupo. En uno de los papeles pone que han ganado 100 euros cada uno/a y en el otro papel pone que se les ha impuesto una sanción de 100 euros. Ambos grupos deberán tratar de memorizar la lista de palabras que aparece en la diapositiva. Cuando terminen deberán escribir las palabras que recuerden de la lista. Se espera que los que han recibido la noticia de que han ganado 100 euros recuerden mejor las palabras y además recuerden más palabras relacionas con emociones/sensaciones agradables: Ganar, placer, risas, esperanza mientras que los que han perdido 100 euros recuerden peor $y$ sobre todo las palabras asociadas con emociones desagradables: dolor, sufrimiento, ruina, enfado.

\section{Actividad 4.5. Tarea}

Objetivo: Analizar las propias emociones y los acontecimientos y sensaciones que las acompañan.

Duración: 10 minutos al día durante una semana.

Materiales: Presentación Sesión 4 (diapositiva 14).

Procedimiento: Durante la próxima semana, estarán atentos/as a cualquier emoción que les surja durante el día y analizar qué puede estar causando la aparición de esa emoción y qué hacen cuando la sienten. Por ejemplo: cuando alguien me falta al respeto siento ira y vergüenza, así que pienso en vengarme y luego le hago una jugarreta a esa persona. Deben traer las reflexiones para la próxima sesión.

\section{Sesión 5. Facilitación emocional. Parte 2.}

Objetivo: Aprender técnicas para facilitar el pensamiento por medio de las emociones (razonamiento y creatividad).

Materiales: Presentación “Sesión 5.”, Facilitación emocional. Parte 2", cuestionario breve de evaluación (Anexo 2), plantilla de observación participante (Anexo 1).

Procedimiento: Para empezar la sesión se pedirá que cada uno/a entregue las notas que haya tomado en la tarea de la sesión anterior durante la última semana, el/la monitor leerá algunos en voz alta y luego preguntará para cada uno de los casos si habría alguna alternativa haciendo uso de sus emociones para influir en su pensamiento más útil para la persona. Haciendo uso de la presentación "Sesión 5.", Facilitación emocional. Parte 2 " se continuará con la explicación de como las emociones influyen en el pensamiento, haciendo alusión ahora a la dificultad de razonamiento y de cambio de perspectiva que puede suceder si las emociones son muy intensas y no las controlamos. Explicando algunas estrategias que pueden ser útiles para que eviten en la medida de lo posible, que esto les suceda. Al finalizar la sesión los/as participantes deben rellenar el cuestionario breve de evaluación (Anexo 2).

Deberán llevarse a cabo las siguientes actividades:

Actividad 5.1. Juego de Rol.

Objetivo: Vivir en primera persona, de manera exagerada, el impacto que tienen las emociones en nuestra capacidad de concentración y 
razonamiento.

Duración: 30 minutos.

Materiales: presentación de la Sesión 5 (Diapositivas 5 y 6).

Procedimiento: Por parejas, uno de los/as participantes va a representar una emoción y el otro/a de sí mismo/a. Lo ideal es hacerlo con todas las emociones, asignaremos una emoción a cada persona e invertirán los papeles cambiando la emoción. La persona que haga de emoción debe, con palabras relacionadas con la emoción distraer a la otra persona de su tarea (leer texto de la diapositiva y responder a las preguntas en una hoja en blanco por escrito). Por ejemplo: Antonio es la ira y Sonia es Sonia. Cuando Sonia trate de responder a las preguntas sobre el texto, Antonio comenzará a hablarle diciéndoles palabras como: estoy enfadada, odio a ese profesor, mi hermano es idiota, se van a enterar... etc. Cada vez que alguno de los/as participantes que hacen de sí mismos/as use una de las técnicas la emoción hablará más bajito, hasta quedarse callada. Para responder a las preguntas deben escribir en un papel las respuestas que crean correctas, una vez que terminen que las lean en voz alta y luego se pondrán las respuestas correctas en la diapositiva para comprobar si han podido concentrarse en la lectura del texto. Una vez finalizada, debatir porque creen que se han distraído, o si no lo han hecho, que nos cuenten cómo lo han hecho para no distraerse.

Actividad 5.2. Video: Emociones creativas.

Objetivo: aprender cómo influyen las emociones en el proceso creativo.

Duración: 10 minutos aprox.

Materiales: Presentación Sesión 5 (diapositiva 8), video

"Emociones creativas" Procedimiento: en la diapositiva, pinchar el enlace para ver el video "Emociones creativas" de la cuenta de YouTube "Margarita Ochoa" y comentarlo con los/as participantes.

Actividad 5.3. A dibujar

Objetivo: Experimentar si su proceso creativo puede ser influenciado por las emociones.

Duración:15 minutos (trabajo en casa durante una semana).

Materiales: presentación de la Sesión 5 (Diapositiva 10).

Procedimiento: Se les pedirá que hagan un dibujo sencillo, y que lo realicen varias veces a lo largo de la próxima semana cuando estén experimentando alguna emoción intensa.

Para en la siguiente sesión comentar si hay diferencias, o no, y por qué creen que es así.

* Sesiones 6 y 7: correspondientes a la habilidad 3 del modelo teórico de Mayer y Salovey (comprensión emocional) y al concepto de empatía contemplado en el modelo de Goleman.

Sesión 6. Comprensión emocional.

Objetivo: Incentivar la activación de mecanismos para la comprensión emocional y reflexionar sobre los comportamientos emocionales propios y de otros/as

Materiales: Presentación "Sesión 6. Comprensión emocional", cuestionario breve de evaluación (Anexo 2), plantilla de observación participante (Anexo 1).

Procedimiento: Al inicio de la sesión se comentará la temática y se explicará brevemente qué va a verse en la sesión. Seguidamente, se hará referencia a algunas nociones muy básicas sobre las bases cerebrales de la empatía, así como la capacidad de las emociones para interferir o potenciar con el funcionamiento de la memoria. Se abordarán los desencadenantes de las mismas. Al final de la sesión se aborda el tema del paso de una emoción a otra y se pregunta los/as participantes experiencias en las que les haya ocurrido esto y se les pregunta por qué creen que sucedió eso. Al finalizar la sesión los/as participantes deben rellenar el cuestionario breve de evaluación (Anexo 2). Así mismo, se harán las siguientes actividades:
Actividad 6.1. Comencemos por lo fácil.

Objetivo: Identificar factores internos o externos que suelen desencadenar emociones en los participantes

Duración: 15 minutos

Materiales: presentación sesión 6 (diapositiva 7), papel y bolígrafo para cada participante.

Procedimiento: Los/as participantes deberán elaborar una lista que contenga al menos dos cosas que les produzca una emoción simple (un evento interno y otro externo). El/a monitor/a leerá las listas en voz alta para ver si coinciden en algunas cosas.

Actividad 6.2. Video: ¿Cómo comprender mejor nuestras emociones?

Objetivo: iniciar el aprendizaje de comprender las propias emociones y las ajenas.

Duración: 20 minutos.

Materiales: presentación sesión 6 (diapositiva 9), vídeo “Cómo comprender mejor nuestras emociones?"

Procedimiento: al llegar a la diapositiva 9 de la presentación 6 se seguirá el enlace web al video "¿Cómo comprender mejor nuestras emociones?" del perfil de YouTube "Miguel Ángel Diaz Escoto".

Actividad 6.3. ¿Por qué esa emoción?

Objetivo: identificar las causas de emociones ajenas.

Duración: 5 minutos.

Materiales: presentación sesión 6 (diapositiva 10).

Procedimiento: cuando llegue la diapositiva 10, deberán leerse las situaciones que aparecen reflejadas en la misma, preguntando en cada ocasión a los/as participantes cual creen que es la causa de la emoción que experimentan los/as protagonistas de los textos. Posteriormente se corregirán las respuestas en la misma diapositiva.

Actividad 6.4. Emociones motivas por...

Objetivo: iniciar el aprendizaje de comprender las propias emociones y las ajenas.

Duración: 10 minutos.

Materiales: presentación sesión 6 (diapositiva 11).

Procedimiento: En la diapositiva 11, deben mirar la imagen y tratar de establecer cuál es la razón para que la persona que se muestra sienta las diferentes emociones aparecen escritas, para luego compartir opiniones sobre las explicaciones dadas.

Sesión 7. Empatía.

Objetivo: Potenciar el conocimiento sobre empatía y activar mecanismos de empatía.

Materiales: Presentación "Sesión 7. Empatía", cuestionario breve de evaluación (Anexo 2), plantilla de observación participante (Anexo 1).

Procedimiento: En esta sesión se comenzará explicando que es la empatía, donde nace, como se trabaja y por qué es importante. Se llevarán a cabo las actividades descritas y al finalizar la sesión los/as participantes deben rellenar el cuestionario breve de evaluación (Anexo 2).

Actividad 7.1. Video: El valor de la empatía y debate.

Objetivo: reflexionar y expresar opiniones sobre qué es la empatía y como se manifiesta.

Duración: 10 minutos

Materiales: presentación de la sesión 7 (diapositiva 9), video “¡El valor de la empatía!”.

Procedimiento: al llegar a la diapositiva $n^{\circ} 9$, se seguirá el enlace para ver el video “¡El valor de la empatía!” de la cuenta de YouTube "Juan lebu Salazar "y se les harán algunas preguntas sobre lo que han visto (mirar las notas de esa diapositiva).

Actividad 7.2. Video: Empatía, signo de grandeza y debate. 
Objetivo: adquirir nociones básicas sobre el concepto de empatía y hacer una reflexión grupal de lo que saben o han aprendido sobre ella.

Duración: 30 minutos.

Materiales: presentación de la sesión 7 (diapositivas 10 y 11) video "Empatía, signo de grandeza $\mid$ Abel Trillini | TEDx paseo Alameda".

Procedimiento: al llegar a la diapositiva 10 se seguirá el enlace al video "Empatía, signo de grandeza | Abel Trillini | TEDx paseo Alameda". Del perfil de YouTube "TEDx Talks". Tras visionar el video tendrán que hacer un debate y responder a las preguntas que aparecen en la diapositiva 11.

Actividad 7.3. Nosotros y ellos.

Objetivo: Experimentar en primera persona una situación en la que el interlocutor no es empático y otra situación en la que sí lo es.

Duración: 15 minutos

Materiales: presentación Sesión 7 (diapositivas 12, 13 y 14).

Procedimiento: Se divide en dos grupos a los/as participantes. Uno de los grupos va a hacer lo que ponga en la diapositiva $13 \mathrm{y}$ el otro grupo deberá hacer lo que pone en la diapositiva 14. Cada grupo sólo podrá leer lo que pone la diapositiva que le corresponda (los azules leerán la 14 y los verdes la 13). Después de 5 minutos deberán cambiar los papeles. Para terminar se les pedirá que hagan una reflexión por escrito como tarea sobre cómo se han sentido experimentando en un papel $u$ otro.

* Sesión 8: correspondiente a la habilidad 4 del modelo teórico de Mayer y Salovey (regulación reflexiva de las emociones).

Sesión 8. Regulación de las emociones.

Objetivo: Aumentar la capacidad de autocontrol de las emociones y hacer una reflexión sobre los comportamientos emocionales propios y de otros/as.

Materiales: Presentación "Sesión 8. Regulación de las emociones", cuestionario breve de evaluación (Anexo 2), plantilla de observación participante (Anexo 1).

Procedimiento: Al inicio de la sesión se leerán las reflexiones hechas por los/as participantes en la sesión anterior. Se les explicará en qué consiste la regulación emocional y la importancia de aprender a regular las emociones. Así mismo, se expondrán y trabajarán 3 técnicas de regulación emocional (difusión de la emoción, centrarse en el interior y verbalización) y se realizarán las actividades reflejadas a continuación. Al finalizar la sesión los/as participantes deben rellenar el cuestionario breve de evaluación (Anexo 2) y el Cuestionario de satisfacción (Anexo 3).

Actividad 8.1. Video: cómo controlar tus emociones y debate

Objetivo: Analizar cuáles son los problemas individuales por falta de regulación emocional y evaluar el grado en que perciben su capacidad de regulación emocional.

Duración: 20 minutos

Materiales: presentación Sesión 8 (diapositiva 9), video

"Inteligencia Emocional •

Cómo Controlar las Emociones"

Procedimiento: cuando aparezca la diapositiva 9, pinchar el enlace para ver el video

Tras visionar el video "Inteligencia Emocional • Cómo Controlar las Emociones" de la cuenta de YouTube "Full Perception" y después tendrán que hacer un debate sobre situaciones en las que han tenido problemas con las emociones, y por qué.

\section{Actividad 8.2. Teatrillo}

Objetivo: Identificar qué emoción, intensidad y técnica de regulación emocional están viendo.

Duración: 25 minutos.

Procedimiento: Deberán representar por parejas un pequeño teatro con mímica en el que uno/a de ellos/as representará una emoción en una intensidad alta o baja, mientras que el otro representará una de las técnicas aprendidas. Los demás deben adivinar la emoción, la intensidad y la técnica utilizada. Por último, se deja un pequeño espacio para compartir la experiencia del programa por parte de los/as participantes, por si quieren contar alguna experiencia relacionada con la IE, etc.

*Evaluación por medio del MSCEIT de los/as participantes. A continuación, en la tabla 1 puede verse un resumen del programa.

Tabla 1

Resumen del programa

\begin{tabular}{lll}
\hline Sesión & Objetivos & Materiales/espacios \\
\hline
\end{tabular}

Comprender el concepto de inteligencia emocional (según el modelo elegido) y las emociones básicas y complejas.

Mejorar el reconocimiento de emociones propias y de otros/as.

Mejorar la expresión de emociones
*Proyector, pantalla, presentación sesión 1.

*Anexos 1 y 2.

*Papel y bolígrafo

*Habitación con sillas

*Proyector, pantalla, presentación sesión 2 .

*Anexos 1, 2 y 5.

* Papel y bolígrafo

*Habitación con sillas
Facilitar la exposición de experiencias personales en presencia de un grupo.
*Proyector, pantalla, presentación sesión 3.

*Anexos 1 y 2.

*Papel y bolígrafo *Habitación con sillas
Actividad 1.1 Y tú ¿qué sientes? Actividad 1.2. Video: El puente: inteligencia emocional" $y$ debate.

Actividad 2.1. ¿Qué sentirías si...?

Actividad 2.2. Adivina qué emoción soy. Actividad 2.3.¿qué estarán sintiendo? Actividad 2.4. Hasta los objetos sienten.

Actividad 3.1. Video: expresión de las emociones.

Actividad 3.2. Expresa lo que sientes y lo que necesitas.

Actividad 3.3. Video:

Lenguaje no verbal.

Actividad 3.4. Video: Las emociones y la voz. 
Tabla 1

Resumen del programa (continuación)

\begin{tabular}{|c|c|c|c|}
\hline Sesión & Objetivos & Materiales/espacios & Actividades \\
\hline 4 & $\begin{array}{l}\text { Aprender técnicas para facilitar el } \\
\text { pensamiento por medio de las emo- } \\
\text { ciones (memoria) }\end{array}$ & $\begin{array}{l}\text { *Proyector, pantalla, presentación sesión } 4 . \\
\text { *Anexos } 1 \text { y } 2 . \\
\text { *Papel y bolígrafo } \\
\text { *Habitación con sillas }\end{array}$ & $\begin{array}{l}\text { Actividad 4.1. Video: } \\
\text { Emoción y atención. Actividad 4.2. Dinámica } \\
\text { Actividad 4.3. Video: El poder de las palabras y } \\
\text { las emociones...” y debate. } \\
\text { Actividad 4.4. Experimento } \\
\text { Actividad 4.5. Tarea }\end{array}$ \\
\hline 5 & $\begin{array}{l}\text { Aprender técnicas para facilitar el } \\
\text { pensamiento por medio de las emo- } \\
\text { ciones (razonamiento y creatividad). }\end{array}$ & $\begin{array}{l}\text { *Proyector, pantalla, presentación sesión } 5 \text {. } \\
\text { *Anexos } 1 \text { y } 2 . \\
\text { *Papel y bolígrafo } \\
\text { *Habitación con sillas }\end{array}$ & $\begin{array}{l}\text { Actividad 5.1. Juego de Rol Actividad 5.2. } \\
\text { Video: } \\
\text { Emociones creativas. } \\
\text { Actividad 5.3. A dibujar. }\end{array}$ \\
\hline 6 & $\begin{array}{l}\text { Incentivar la activación de mecanismos } \\
\text { para la comprensión emocional. Hacer } \\
\text { una reflexión sobre los comportamien- } \\
\text { tos emocionales propios y de otros/as }\end{array}$ & $\begin{array}{l}\text { *Proyector, pantalla, presentación sesión } 6 . \\
\text { *Anexos } 1 \text { y } 2 . \\
\text { *Papel y bolígrafo } \\
\text { *Habitación con sillas }\end{array}$ & $\begin{array}{l}\text { Actividad 6.1. Comencemos por lo fácil. } \\
\text { Actividad 6.2. Video: ¿Cómo comprender mejor } \\
\text { nuestras emociones? } \\
\text { Actividad 6.3.¿Por qué esa emoción? } \\
\text { Actividad 6.4. Emociones motivas por... }\end{array}$ \\
\hline 7 & $\begin{array}{l}\text { Potenciar el conocimiento sobre empa- } \\
\text { tía y activar mecanismos de empatía. }\end{array}$ & $\begin{array}{l}\text { *Proyector, pantalla, presentación sesión } 7 . \\
\text { *Anexos } 1 \text { y } 2 . \\
\text { *Papel y bolígrafo } \\
\text { *Habitación con sillas }\end{array}$ & $\begin{array}{l}\text { Actividad 7.1. Video: } \text { El } \\
\text { valor de la empatía y debate. } \\
\text { Actividad 7.2. Video: Empatía, signo de } \\
\text { grandeza y debate } \\
\text { Actividad 7.3. Nosotros y ellos }\end{array}$ \\
\hline 8 & $\begin{array}{l}\text { Aumentar la capacidad de autocontrol } \\
\text { de las emociones y hacer una } \\
\text { reflexión sobre los comportamientos } \\
\text { emocionales propios y de otros/as. }\end{array}$ & $\begin{array}{l}\text { *Proyector, pantalla, presentación sesión } 8 . \\
\text { *Anexos } 1,2 \text { y } 3 \text {. } \\
\text { *Papel y bolígrafo } \\
\text { *Habitación con sillas. }\end{array}$ & $\begin{array}{l}\text { Actividad 8.1. Video: cómo controlar tus emo- } \\
\text { ciones y debate } \\
\text { Actividad 8.2. Teatrillo. }\end{array}$ \\
\hline
\end{tabular}

\section{Evaluación del programa}

Para la medida del nivel de inteligencia emocional, se hará uso de la adaptación al castellano del MSCEIT v.2.0 realizada por Extremera et al. (2002) ya que responde al marco teórico del que se va a servir el presente programa para guiar la intervención. Este instrumento debe aplicarse antes de la realización del programa para obtener una medida comparativa con respecto a la que se obtenga cuando finalice el programa y se aplique nuevamente este instrumento.

Por otro lado, aquellas personas que imparten el programa y que, por tanto, viven en primera persona los problemas o limitaciones que pueden surgir en su aplicación, por lo que es muy recomendable que rellenen el cuestionario final para la mejora del programa (Anexo 4) con la intención de recopilar información para futuras mejoras en el presente programa o en otros diseños similares.

Así mismo, debe hacerse uso de las respuestas recogidas en los cuestionarios breves de evaluación (Anexo 2) de cada sesión como indicadoras del alcance que han tenido las sesiones a nivel individual y el grado de adquisición de los contenidos impartidos a nivel teórico de las sesiones.

Estas respuestas deberán tenerse en cuenta también durante el desarrollo del programa como indicadores del grado de interés que muestran los/as participantes en los contenidos y pueden ayudar a plantear posibles cambios en las dinámicas del programa en caso de que no haya adherencia a las sesiones.

Por último, deberá hacerse uso de las respuestas obtenidas en el cuestionario de satisfacción (Anexo 3) como indicador cualitativo del grado de utilidad percibida por los/as participantes del programa.

\section{Conclusiones}

Este trabajo ha tratado de ofrecer una idea general de la Inteligencia Emocional (IE), sus marcos teóricos y su relación con otras variables para posteriormente, en este mismo manuscrito, se desarrolle una propuesta innovadora de un programa de intervención destinado a menores infractores.

Como se ha visto, la IE podría ser una buena piedra angular desde la que abordar la intervención con menores infractores. De forma que, haciendo uso de bases empíricas y modelos teóricos con fundamentación, es posible crear un programa que recoja los objetivos más importantes que deben alcanzar estos/as jóvenes en lo que a habilidades emocionales se refiere, permitiendo una mayor probabilidad de aumentar los factores de protección mientras están cumpliendo sus medidas judiciales.

Durante el cumplimiento de las medidas judiciales, y como recoge la Ley Orgánica 5/2000, sobre todo en medidas en régimen de internamiento, es importante que los/as jóvenes adquieran los recursos de competencia social que les permitan comportarse de una forma responsable dentro de la sociedad. Un programa de intervención con las características que se han presentado en este trabajo permitirá desarrollar al menos una parte de esos recursos en el ámbito emocional 
y probablemente contribuirán a la reinserción y a la mejora durante el periodo de cumplimiento de la medida.

Si bien la IE no es la única competencia que debe ser trabajada, debido a que existen gran número de factores de protección en los que puede realizarse una intervención para la mejora de las competencias socio-emocionales de este segmento de la población, se ha visto reflejada durante el presente documento, la acción facilitadora que puede ejercer el desarrollo de la IE en la trayectoria delictiva de los/as menores.

Se ha desarrollado un programa que utiliza las nuevas tecnologías como soporte principal para el material de trabajo y la base teórica desarrollada en los modelos de Mayer y Salovey (1997) y Goleman (1996) para cumplir con el objetivo principal de diseñar un programa innovador.

El soporte digital es una facilidad a la hora de adquirir el material para quienes deban llevar a cabo el programa, puesto que no requiere de más acciones por su parte que la de reproducir las sesiones en un soporte adecuado y razonablemente accesible para la mayoría de las instituciones públicas y privadas que tengan potestad para impartirlo. Además, se adapta perfectamente a la recomendación de las agendas digitales de la Unión Europea

(Parlamento europeo, 2020) y de España (Ministerio de Asuntos Económicos y Transformación Digital, 2020).

Para alcanzar los objetivos propuestos por el programa, se han elaborado un total de 27 actividades que contribuyen a que los contenidos sean experienciales además de teóricos, con intención de facilitar la asimilación de los conocimientos por medio de la experiencia. Cada una de ellas contribuye a conseguir los objetivos de las sesiones, y por tanto a conseguir el objetivo general.

Introduciendo recursos virtuales como los videos o las presentaciones en formato Power Point, se persigue que esta tecnología, que ya forma parte del proceso de socialización en los/as jóvenes (Peirone, 2019), sirva de catalizador para un aprendizaje más fácil y significativo.

Por último, cabe destacar que el programa debe ser revisado cada vez que se imparta, haciendo uso de los registros que se han dispuesto como instrumentos del programa, para futuras mejoras en el mismo. Tratando de establecer, además, si es necesario incluir otros constructos en el programa, que estén relacionados con la IE y que puedan fortalecer el impacto positivo en el aprendizaje por parte de los/as participantes.

\section{Referencias}

American Management Association (2012). Leading with Emotional Intelligence. AMA.

Avilés, J.M. (2005). Bullying: el Maltrato entre Iguales. Agresores, Victimas y Testigos en la Escuela. Amarú.

Barchard, K. A. (2003). Does Emotional Intelligence Assist in the Prediction of Academic Success? Educational and Psychological Measurement, 63(5), 840-858. https:// doi.org/10.1177/0013164403251333

Bar-On, R. (1997). The emotional quotient inventory (EQ-I): A test emotional intelligence. Multi-Health Systems.

Bar-On, R. (2002). Bar-On Emotional Intelligence-Inventory: Short. Multi-Health Systems.

Bar-On, R. (2006). The Bar-On Model of Emotional-Social Intelligence (ESI). Psicothema, 18, 124-131.

Bar-On, R., y Parker J. (2000). Emotional and Social Intelligence: Insights from the Emotional Quotient Inventory (EQ-i). In R. BarOn y J.D.A. Parker (Eds.), Handbook of Emotional Intelligence. Jossey-Bass.
Berrios Martos, M. P., Augusto Landa, J. M., y Aguilar Luzón, M. D. C. (2006). Inteligencia emocional percibida y satisfacción laboral en contextos hospitalarios: Un estudio exploratorio con profesionales de enfermería. Index de Enfermería, 15(54), 30-34.

Bisquerra, R. (2000). Educación Emocional y Bienestar. Praxis.

Buil-Legaz, P., Burón-Álvarez, J.J., y Bembibre, J. (2019). Perfil Sociodemográfico y Delictivo de los Menores Infractores en Medio Abierto en Granada de 2014 a 2017.

Análisis Descriptivo y Evolución. Anuario de Psicología Jurídica, 29(1) 61 -68. https://doi.org/10.5093/apj2019a2

Capdevila, M., Ferrer, M., y Luque, E. (2005). La Reincidencia en el Delito en la Justicia de Menores. Centre d'estudis Jurídics i formació especialitzada. Generalitat de Catalunya.

Caruso, D., y Salovey P. (2005). El Directivo Emocionalmente Inteligente. La Inteligencia Emocional en la Empresa. Algaba.

Cera, E., Almagro, B.J., Conde, C., y Sáenz-López, P. (2015). Inteligencia Emocional y Motivación en Educación Física en Secundaria. RETOS. Nuevas Tendencias en Educación Física, Deporte y Recreación, 1 (27) $8-13$.

Ciarrochi, J., Chan, A., y Bajgar, J. (2001). Measuring Emotional Intelligence in Adolescents. Personality and Individual Differences, 31(7), 1105-1119. https://doi.org/10.1016/s0191-8869(00)00207-5

Coleman, J., y Hendry, L. (2003). Psicología de la Adolescencia. Morata.

Delgado-Gómez, M.S., Gómez-Díaz, M., Gómez-Sánchez, R., y RecheGarcía, C. (2019). Relationship between Emotional Intelligence and Psychopathological Risk in University Students. Formación universitaria, 12(3), 39-46. https://dx.doi.org/10.4067/S0718$\underline{50062019000300039}$

Díaz, E., Jiménez, V., Fernández, A., Zabala, A., y Saez, I. (2020). Entrenamiento de la Inteligencia Emocional en el Caso de una Adolescente Víctima de Maltrato Infantil, Revista de Psicología Clínica con Niños y Adolescentes, 4(1),17-24.

Elena Emociones Básicas. (13 de diciembre de 2016). 7 beneficios de expresar las emociones. [Archivo de video]. YouTube. https:// www.youtube.com/watch? $\mathrm{v}=$ SoOzoLmQn14

Extremera, N., y Fernández-Berrocal, P. (2002). Cuestionario MSCEIT (Versión española 2.0) de Mayer, Salovey y Caruso. Multi-Health Systems Publishers.

Extremera, N., Fernández-Berrocal, P., y Salovey, P. (2006). Spanish Version of the MayerSalovey-Caruso Emotional Intelligence Test (MSCEIT). Version 2.0: Reliabilities, Age and Gender Differences. Psicothema, 18, 42-48.

Extremera, N., Ruiz-Aranda, D., Pineda-Galán, C., y Salguero, J.M. (2011). Emotional Intelligence and its Relation with Hedonic and Eudaimonic Well-Being: A Prospective Study. Personality and Individual Differences, 51, (1) 11-16. http://dx.doi.org/10.1016/j.paid.2011.02.029

Fernández-Berrocal, P., y Extremera, N. (2009). La Inteligencia Emocional y el Estudio de la Felicidad. Revista Interuniversitaria de Formación del Profesorado, 66, 85-103.

Fernández-Berrocal, P., Extremera, N., y Ramos, N. (2004). Validity and Reliability of the Spanish Modified Version of the Trait Meta-Mood Scale. Psychological Reports, 94,

751-755. https://doi.org/10.2466/pr0.94.3.751-755

Full Perception. (8 de julio de 2017). Inteligencia Emocional • Cómo Controlar las Emociones. [Archivo de video]. YouTube. https:// www.youtube.com/watch? $\mathrm{v}=\mathrm{se} 62 \mathrm{UwCxUrI}$

García-Sancho, E., Salguero, J.M., y Fernández-Berrocal, P. (2014). Relationship between Emotional Intelligence and Aggression: A Systematic Review. Aggression and Violent Behavior, 19 (5), 584-591. https:// doi.org/10.1016/j.avb.2014.07.007

Gázquez, J., Pérez-Fuentes, M., Díaz Herrero, Á., García-Fernández, J., e 
Inglés, C. (2015). Perfiles de Inteligencia Emocional y Conducta Social en Adolescentes Españoles. Psicología Conductual, 23 (1), 141-160. https://doi.org/10.11144/Javeriana.upsy18-4.ieap

Goleman, D. (1996). Inteligencia Emocional. Kairós.

Goleman, D. (1999). La Práctica de la Inteligencia Emocional. Kairós.

Goleman, D. (2011). Leadership: The Power of Emotional Intelligence. AMA.

Hemisferio Derecho. (20 de marzo de 2018). Lenguaje NO VERBAL - La Guía de las MANOS. [Archivo de video]. YouTube. https:// www.youtube.com/watch?v=Ti2JZfKw9cA

JOHANNA BIJALBA (7 de enero de 2016). El Puente: Inteligencia Emocional. [Archivo de video]. YouTube. https://www.youtube.com/ watch? $\mathrm{v}=\mathrm{S} 2 \mathrm{seE} 2 \mathrm{JAVB} 0$

Juan lebu salazar. (25 de octubre de 2017). EL VALOR DE LA EMPATÍA!!! [Archivo de video]. YouTube. https://www.youtube.com/watch? $\mathrm{v}=4 \mathrm{Hgmfkg}-\mathrm{UTk}$

Ley Orgánica 5/2000, de 12 de enero, reguladora de la responsabilidad penal de los menores. Boletín Oficial del Estado, 11, 13 de enero de 2000, https://www.boe.es/eli/es/lo/2000/01/12/5/con

López-Zafra, E., Pulido, M., y Berrios, P. (2014). EQ-i Versión Corta (EQiC). Adaptación y validación al español del EQ-i en universitarios. Boletin de Psicología, 110, 21-36.

Marta Pinillos Manrique de Lara. (6 de mayo de 2019). Cómo influyen las emociones en nuestro tono de voz. [Archivo de video]. YouTube. https://www.youtube.com/watch?v=Ti2JZfKw9cA

Margarita Ochoa. Emociones creativas. [Archivo de video]. YouTube. https://www.youtube.com/watch? $\mathrm{v}=\mathrm{G} 01 \mathrm{Bcr} 8 \mathrm{KHOw}$

MÁGICA-Mente. (7 de noviembre de 2017). El PODER DE LAS PALABRAS y de las EMOCIONES - Experimento del Arroz. [Archivo de video]. YouTube. https://www.youtube.com/watch? $\mathrm{v}=$ SYPkmMdwqK0

Mayer, J.D., y Salovey, P. (1993). The Intelligence of Emotional Intelligence. Intelligence 17(4) 433-442.

Mayer, J.D., y Salovey, P. (1997) What is Emotional Intelligence? In P. Salovey y D. Sluyter (Eds.): Emotional Development and Emotional Intelligence: Educational Applications (pp. 3-31). Basic Books.

Mayer, J. D., Salovey, P., y Caruso, D. (2000). Models of Emotional Intelligence. In R. J. Sternberg (Ed.), Handbook of intelligence (p. 396-420). Cambridge University Press. https://doi.org/10.1017/ CBO9780511807947.019

Mayer, J.D., Salovey, P., y Caruso, D.R. (2002). Mayer-Salovey-Caruso Emotional Intelligence Test (MSCEIT), Versión 2.0. Multi-Health Systems.

Miguel Angel Diaz Escoto. (18 de agosto de 2016). La emoción condiciona la atención [Inteligencia Emocional]. [Archivo de video]. YouTube. https://www.youtube.com/watch?v=JgGzlq1KJYM

Miguel Angel Diaz Escoto. (20 de abril de 2019). ¿Cómo comprender mejor nuestras emociones?. [Archivo de video]. YouTube. https:// www.youtube.com/watch?v=KKuLQq0XmjI

Ministerio de Asuntos Económicos y Transformación Digital, (2020). Planes específicos.

Noguera, J. M. S., Aranda, D. R., Martín, R. P., Berrocal, P. F., Pacheco, N. E., y González, R. C. (2014). Programa INTEMO. Guía para mejorar la inteligencia emocional de los adolescentes. Ediciones Pirámide.

Redondo, S., Martínez, A., y Andrés, A. (2011). Factores de Éxito Asociados a los Programas de Intervención con Menores Infractores. Ministerio de Sanidad, Política Social e Igualdad.

Parlamento Europeo (2020). Fichas temáticas sobre la Unión Europea.

Peirone, F. (2019). El saber tecnológico. De saber experto a experiencia social. Virtualidad, Educación y Ciencia, 10(18), 66-80.

Ponce González, N. (2016). Evaluación de un Programa de Intervención de
Inteligencia Emocional: un Estudio Comparado entre España y México (Tesis doctoral) Universidad de Granada. Recuperado de https://hera.ugr.es/tesisugr/26391582.pdf

Ramos N. S., Enríquez, H., y Recondo, O. (2012). Practica Inteligencia Emocional Plena .Kairós

Repetto, E., Pena, M., y Lozano, S. (2007). El Programa de Competencias Socio-emocionales (POCOSE). XXI. Revista De Educación, 9 , 35-41.

Romero, N.A.R., Guajardo, J.G., y Nava, J.M.F. (2017). Competencias Socioemocionales como Predictoras de Conductas Prosociales y Clima Escolar Positivo en Adolescentes. Revista Interuniversitaria de Formación del Profesorado, 31.1(88), 77-90.

Salguero, J.M., Fernández-Berrocal, P., Ruiz-Aranda, D., Castillo, R., y Palomera, R. (2011). Inteligencia Emocional y Ajuste Psicosocial en la Adolescencia: El Papel de la Percepción Emocional. European Journal of Education and Psychology, 4(2), 143152.

Salovey, P., y Mayer, J.D. (1990). Emotional Intelligence. Imagination, Cognition and Personality, 9(3), 185-211. https://doi.org/10.2190/ DUGG-P24E-52WK-6CDG

Salovey, P., Mayer, J.D., Goldman, S.L., Turvey, C., y Palfai, T.P. (1995). Emotional Atention, Clarity, and Repair: Exploring Emotional Intelligence Using the Trait MetaMood Scale.En J. W. Pennebaker (Ed.): Emotion, Disclosure and Health (p. 125151): American Psychological Association.

Sanabria, A.M., y Uribe Rodríguez, A.F. (2010). Factores Psicosociales de Riesgo Asociados a Conductas Problemáticas en Jóvenes Infractores y No Infractores. Diversitas: Perspectivas en Psicologia, 6(2), 257-274.

Sánchez, A. (2009). El Valor de la Inteligencia Emocional. Ser Emocionalmente Inteligente para Hallar la Felicidad y Mejorar la Calidad de Vida. Formación Alcalá

Sánchez-Teruel, D., y Robles-Bello, M.A. (2018). Instrumentos de Evaluación en Inteligencia Emocional: Una Revisión Sistemática Cuantitativa. Perspectiva Educacional, 57(2), 27-50. http:// dx.doi.org/10.4151/07189729-vol.57-iss.2-art.712

TEDx Talks. (13 de marzo de 2018). Empatía, signo de grandeza | Abel Trillini $\mid$ TEDxPaseoAlameda. [Archivo de video]. YouTube. https://www.youtube.com/watch?v=FoLyQo-I-20

Tere Gutierrez (15 de noviembre de 2016). Presentación emociones [Archivo de video]. YouTube. https://www.youtube.com/watch? $\mathrm{v}=\mathrm{b} 37 \mathrm{MocJP} 3 \mathrm{dI}$

Vázquez, M.J., Fariña, F., Arce, R., y Novo, M (2003). Principales Factores de Riesgo y Protectores del Comportamiento Agresivo y Antisocial. En R. Arce y F. Fariña (Eds.), Avances entorno al Comportamiento Antisocial, Evaluación y Tratamiento (pp. 103139). Ministerio de Trabajo y Asuntos Sociales.

Vilariño, M., Amado, B.G., y Alves, C. (2013). Menores Infractores: Un Estudio de Campo de los Factores de Riesgo. Anuario de Psicología Jurídica, 23(1), 39-45. https://doi.org/10.5093/aj2013a7

Zavala, M., Valadez, M., y Vargas, M. (2008). Emotional Intelligence and Social Skills in Adolescents with High Social Acceptance. Electronic Journal of Research in Educational Psychology, 6(2), 319-338. 